\title{
Pricing of a European Call Option Under a Local Volatility Interbank Offered Rate Model
}

\author{
Winnie Mbusiro Chacha, Caroline Njenga, Wilson Mahera \\ Pan African University, Institute of Basic Sciences, Nairobi, Kenya \\ Email address: \\ winniemchacha@gmail.com (W. M. Chacha)

\section{To cite this article:} \\ Winnie Mbusiro Chacha, Caroline Njenga, Wilson Mahera. Pricing of a European Call Option Under a Local Volatility Interbank Offered \\ Rate Model. American Journal of Theoretical and Applied Statistics. Vol. 7, No. 2, 2018, pp. 80-84. doi: 10.11648/j.ajtas.20180702.14
}

Received: February 1, 2018; Accepted: February 24, 2018; Published: March 22, 2018

\begin{abstract}
This research seeks to give insight on how advances in developed money markets can be reflected towards the establishment of derivatives markets in under developed and developing financial markets. The dynamics of the London interbank offered rate, for the developed financial market and the Kenyan interbank offered rate, for the developing financial markets, are compared. For the period between 2013-2015, both interest rates are found to have the same underlying dynamics. A European caplet is priced using the local volatility interbank offered rate model. The local volatility model is used as it captures the volatility smiles more efficiently in one sweep. Thereafter, the local volatility interbank offered rate model is formulated and used to price the European caplet for the developing markets.
\end{abstract}

Keywords: Call Option, Kenyan IBOR, LIBOR

\section{Introduction}

The Interbank Offered Rate (IBOR) is the average interbank interest rate at which banks in a money market are prepared to lend money to each other. This interest rate is important to both professional and private individuals as it is the benchmark rate for financial instruments traded on the financial markets. Examples of the IBORS include the London IBOR, Kenyan IBOR, and European IBOR.

An option is a financial derivative that gives the holder the right to buy or sell an underlying security at a specified price, at a specified future date. The underlying asset could be interest rates, stocks, exchange rates or any financial variable of interest to a researcher. An option's price depends on the volatility of the underlying asset price, which in this research is the Kenyan IBOR interest rate. This volatility affects the distribution of the assets at expiry and hence the expected return from the option. Investors invest in options for the purposes of hedging, speculating or an existing arbitrage opportunity.

The derivatives market in Kenya is under developed. The market is inactive due to factors such as lack of awareness among the potential local and foreign investors, inadequate risk management techniques and poor legislation with regards to derivatives trading and taxation. This research prices an interest rate derivative for the Kenyan money market thereby introducing a new investment security for the local and foreign investors.

Traditionally, the Black-Scholes option pricing formula introduced by Black \& Scholes (1973) has been used as the benchmark to price the European vanilla options. Black-

Scholes (BS) model exhibits strong pricing biases across both maturity and moneyness. One of the biases is the assumption that the distribution of the underlying stock is log- normal with known mean and variance. This is not true since the underlying stock has a high kurtosis and the assumption on constant variance is somewhat dubious as there are long-term observed features of the implied volatility such as volatility smiles and skews.

It is important to consider a model that takes into consideration the varying volatility of the underlying security under consideration. There are a number of models that take into account the volatility skews and smiles. These models include the stochastic volatility models, jump models, variance gamma models and local volatility models.

Some of the stochastic volatility models introduced were by Hull \& White (1987), Stein \& Stein (1991) and Heston (1993). Merton (1976) introduces the likelihood of jumps in the stochastic process for the underlying asset price that allows for the existence of volatility skew. The variance 
gamma process for the stock price was introduced by Madan et al. (1998). This process generalizes the Brownian motion and allows for the existence of skewness and kurtosis in the return distribution.

Stochastic volatility models are computationally complex and they pose an extreme difficulty of fitting parameters to the current prices of interest rate options. Researchers found a simpler way of pricing exotic options consistently with the volatility skew. Dupire et al. (1994) introduced the concept of local volatility model whereby they noticed that under risk neutrality, there existed a unique diffusion process consistent with this distributions.

A local volatility model, is one that treats volatility as a deterministic function of both the current asset level and time. In other words, local volatilities represent some kind of average over all possible instantaneous volatilities in a stochastic volatility world, (Berestycki et al., 2002). As such, a local volatility model is a generalization of the BlackScholes model. The local volatility model allows for the simplification of assumptions that allowing practitioners to price options consistently with the known prices of vanilla options.

The local volatility type modelling captures the surface of the implied volatilities more precisely than other approaches, (Henry-Labordere, 2009). Of importance to note is that the local volatility framework is an arbitrage free and risk neutral valuation framework. The local volatility framework is adopted to determine the European call option price with the underlying asset as the IBOR interest rate. With the introduction of derivatives market at the Nairobi Securities Exchange, an accurate pricing model for the call options on the IBOR rates will offer the best investment option for both foreign and local investors.

\section{Literature Review}

The development of the local volatility model by Dupire et al. (1994) was a major advancement in handling of volatility smiles. The local volatility models are arbitrage-free, selfconsistent and can easily be calibrated to match the observed market smiles, (Henry-Labordere, 2009). Another development in the capturing of interest rate derivatives smile in a local volatility framework was by Andersen \& Andreasen (2000). The Libor market model is extended to markets with volatility skews in observable option prices. They expanded the family of forward rate in order to develop a special case of the local volatility models in order to capture the skews and smiles. This is an alternative methodology to pricing of interest rate derivatives in the local volatility framework.

The Dupire type local volatility model requires one to obtain differentiation against maturity. The traditional continuous stochastic process that has been used in interest rate vanilla pricing cannot allow for differentiation against maturity. Zhu \& Qu (2016) creates a spot process that allows one to differentiate against maturity and hence strip local volatility to price caps.
Zhu \& Qu (2016) present a simpler and practical model that handles the interest rate smile. The model allows direct Dupire-type local volatility stripping in the asset class of interest rates. The model also formulates a backward-pricing partial differential equation using a numeraire deflated value, which can be used to price suitable path-dependent interest rate derivatives with a smile. This self-contained smile model possesses all the good features of a Dupire-type local volatility model, including numerical simplicity and efficiency. This is the approach that is employed in this research due to its simplicity and efficiency.

\section{Methodology}

Consider a probability space $(\Omega, F, F, P)$ where the filtration $\mathrm{F}=\left(F_{t}\right)_{t \in[0, T]}$ satisfies the usual conditions of a filtration and $T$ denotes a finite time horizon with a discrete tenor structure. Let $W=\left(W_{t}\right)_{t \in[0, T]}$ denotes a standard Brownian motion.

Denote the forward martingale measure by $\left(P_{t}\right) t \in T$ and where the corresponding zero coupon bond $B($.$) acts as a$ numeraire for each forward measure. All the forward measures are assumed to be equivalent to the measure $\mathrm{P}$. The forward IBOR rates over the future accrual period $[t, T+$ $\delta$ ] observed at time $t$, is set to satisfy the equation

$$
1+\delta L(t, T)=\frac{B(t, T)}{B(t, T+\delta)}=F(t, T, T+\delta)
$$

Which is also expressed as

$$
L(t, T)=\frac{B(t, T)-B(t, T+\delta)}{B(t, T+\delta)}
$$

The dynamics of the system of IBOR rates under the forward martingale measure is given by:

$$
d L(t)=\lambda(T, T+\delta)(t) \cdot d W T, T+\delta(t)
$$

In relation to equation (1) when using the local volatility type model, the differentiation against maturity cannot be obtained thus a spot process introduced by Zhu \& Qu (2016) that allows differentiation against maturity and local volatility stripping is employed.

Research follows the approach of Zhu \& Qu (2016) in determining the dynamics of the rolling IBOR rates. Denoting the time- $t$ rolling IBOR as $L_{t, t+\delta(t)}$ and the zero coupon bond price as $B(t, t+\delta)$, the spot process of the rolling IBOR is assumed to follow the normal process:

$$
d L_{t, t+\delta(t)}=\mu_{t, t+\delta(t)} d t+\sigma_{(t, t+\delta)}(t) \cdot d W_{t, t+\delta}(t)
$$

where $\mu_{t, t+\delta(t)}$ is the drift, $\sigma_{(t, t+\delta)}(t)$ is the diffusion coefficient term and $W_{t, t+\delta}(t)$ is the standard Brownian motion under the spot measure $Q^{\text {spot }}$ associated with the local numeraire $B(t, t+\delta)$.

Zhu \& Qu (2016) find that the drift term $\mu_{t, t+\delta}(t)$ is given by: 


$$
\mu_{t, t+\delta(t)}=-\frac{\partial\left(\frac{B(t, T+\delta)}{B(t, t+\delta)} L_{T, T+\delta(t)}-L_{t, t+\delta(t)}\right.}{\partial t}
$$

The drift equation enables the derivation of the pricing formulae for interest rate vanillas under the rolling IBOR spot process.

The cap pricing formula earlier derived under the spot IBOR process can be used for local volatility stripping as it permits differentiation against the option maturity allowing the application of the Dupire-type local volatility.

The normal local volatility can be stripped directly from the implied volatility. Using the Bachelier call, the normal local volatility stripping formula is given by:

$$
\sigma(T, K)=\sqrt{\frac{2 \frac{\partial \sigma_{1}}{\partial T}+\frac{\sigma_{1}}{T}+2 \mu(T) \frac{\partial \sigma_{1}}{\partial K}}{\frac{1}{\sigma_{1} T}\left(1+\frac{(F(T)-K) \partial \sigma_{1}}{\sigma_{1}}\right)^{2}+\frac{\partial^{2} \sigma_{1}}{\partial K^{2}}}}
$$

Applying the Feyman-Kac theorem and the fact that $L_{t, t+\delta}$ $(t)$ follows the local volatility normal process, Zhu \& Qu (2016) arrive at the following Kolmogorov backward PDE:

$$
\frac{\partial P}{\partial t}+\mu(t) \frac{\partial P}{\partial L}+\frac{1}{2} \sigma^{2}(t, L) \frac{\partial^{2} P}{\partial L^{2}}=0
$$

The above backward PDE provides an efficient way to price the interest rate call option in the presence of a volatility smile.

\section{Results and Discussion}

The dataset used is London Interbank Offered Rate (LIBOR) for developing markets and Kenyan Interbank Offered Rate for developed markets. The period of study is 2013-2015. One of the objectives of the research was to determine if the dynamics of the developing markets and of the developed markets IBOR rates are similar.

\section{A 2013 to 2015 plot of the Kenyan IBOR rates}

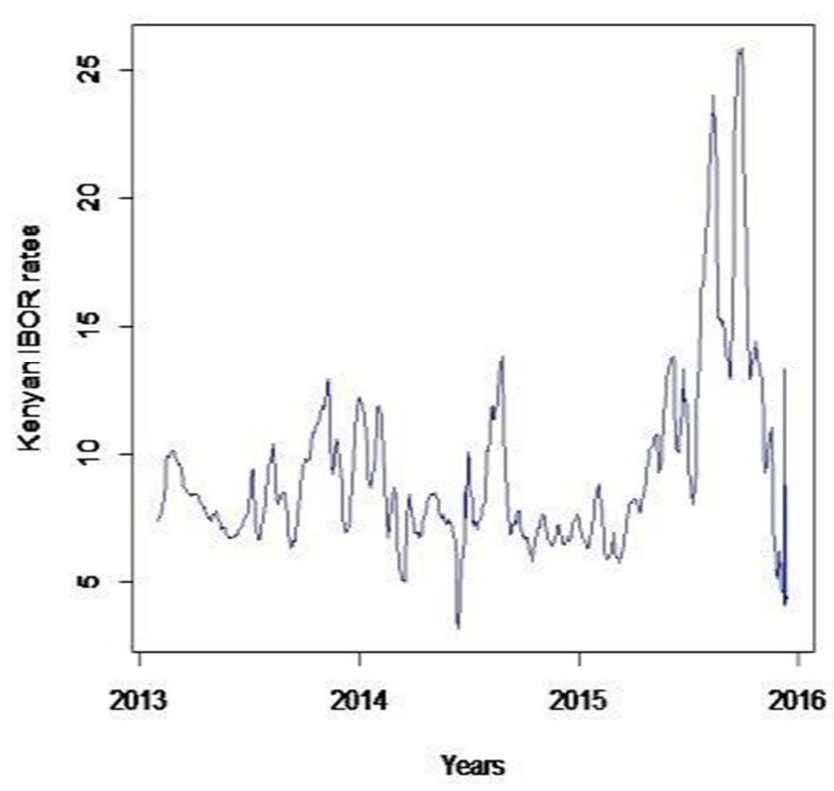

\section{A 2013 to 2015 plot of the London IBOR rates}

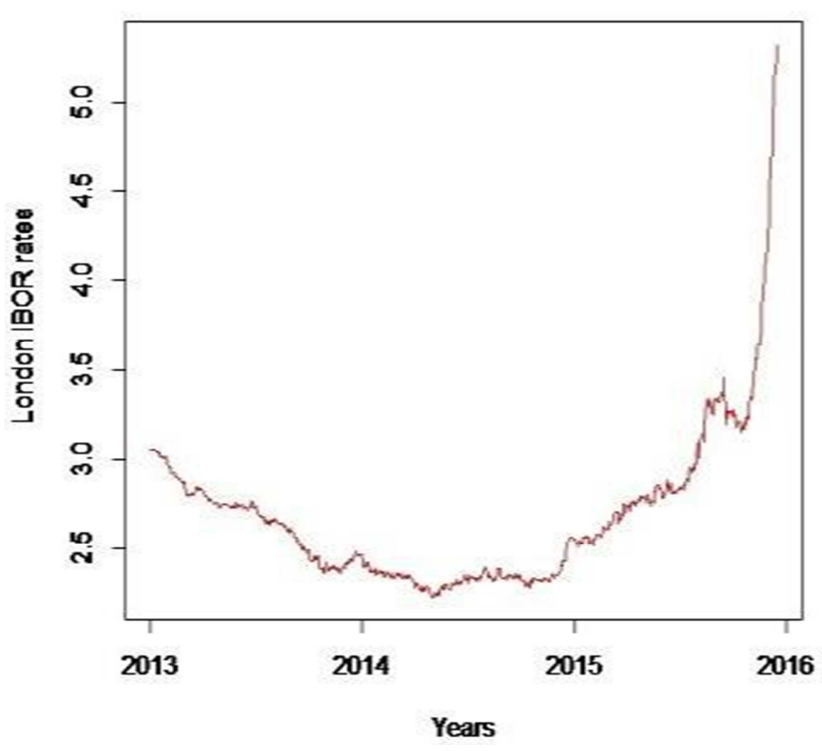

Figure 1. Kenyan IBOR and London IBOR Rates.

A comparison of the patterns and behaviors of the LIBOR and Kenyan IBOR over time.

From Figure 1, it is observed that the Kenyan IBOR rate is more volatile and changes more dramatically than the London IBOR. This shows an option where the underlying security is the Kenyan IBOR is a riskier investment than one which the underlying security is the LIBOR. The LIBOR rates is more stable than the Kenyan IBOR. This explains why for interest rate derivatives in developed financial markets the underlying asset is mostly the LIBOR.

Table 1. Descriptive Statistics of the IBORS used in the empirical study.

\begin{tabular}{lll}
\hline Descriptive Statistics & Kenyan IBOR & London IBOR \\
\hline Mean & 9.293 & 2.683 \\
Median & 8.229 & 2.607 \\
Stdev & 3.633 & 0.441 \\
Skewness & 2.225 & 2.482 \\
Kurtosis & 6.161 & 9.300 \\
\hline
\end{tabular}

These descriptive statistics show the summaries difference between the developing markets IBOR rates and developed markets IBOR rates

The characteristic of volatility clustering is more evident on the Kenyan IBOR than the LIBOR. This is because the large changes in the Kenyan IBOR are followed by large changes and the small changes are followed by small changes. Moreover, in terms of the scale of the datasets, the Kenyan IBOR varies over a larger scale of 0-25 while the LIBOR varies over the scale of $0-5$. This shows that the Kenyan IBOR is more volatile.

All these show that an interest rate derivative on Kenyan IBOR is a very risky asset for investors. This explains why there are no interest rate derivatives trading in developing markets and the need to have a model that can accurately price these derivatives for a developing market. The descriptive statistics indicate that both IBOR rates are not normally distributed. This is shown by the kurtosis of the two 
datasets which is greater than 3 indicating heavy tail. Moreover, both datasets are asymmetrical since they both exhibit a positive skew. This shows that the two data sets are from a similar distribution which is a heavy tailed one. This indicates similarity between the Kenyan IBOR and London IBOR rates.

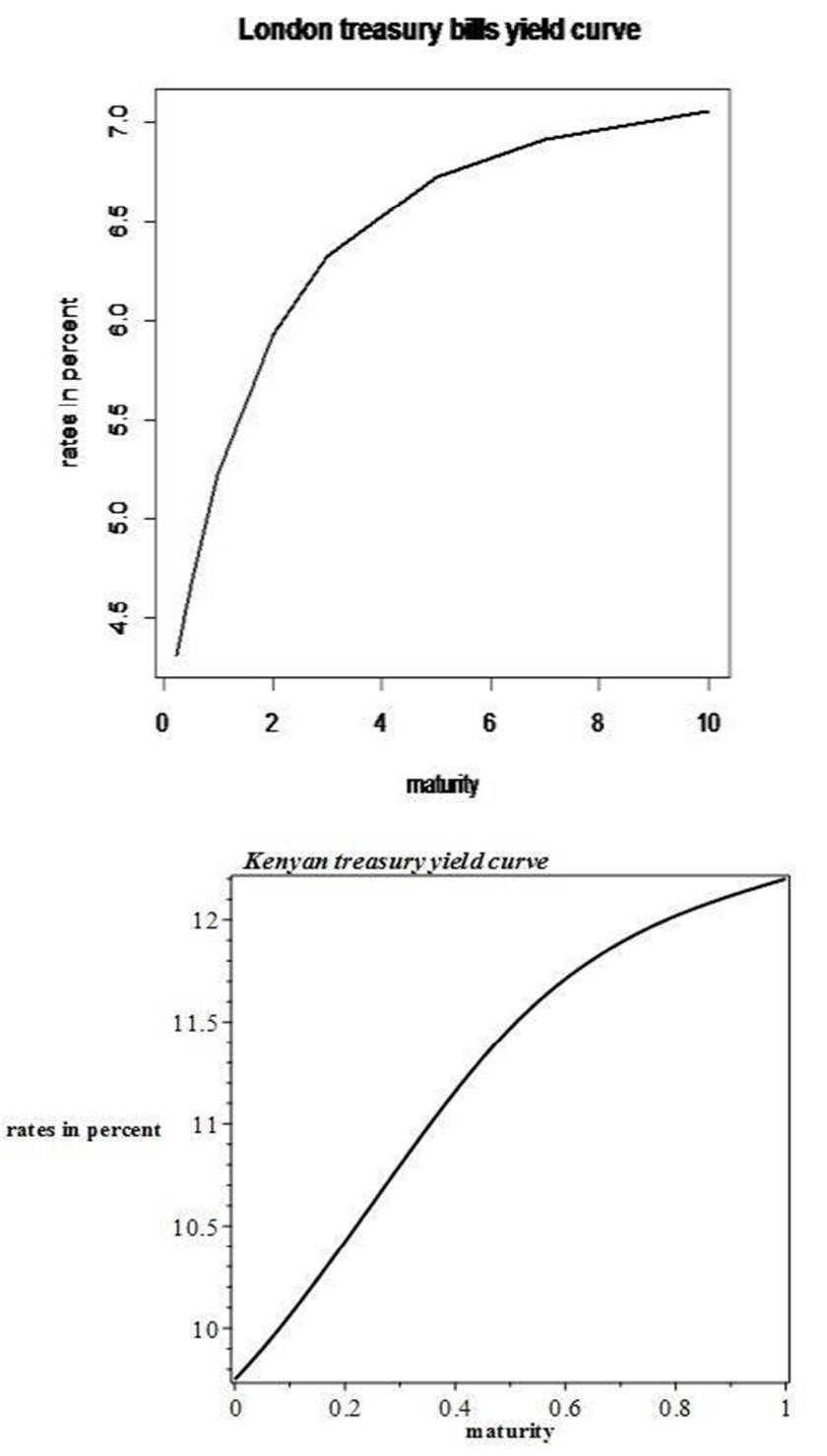

Figure 2. Kenyan IBOR and London IBOR yield curves (zero rate).

This shows the comparison of the developed market yield curve and the developing market yield curve.

Figure 2 of today's yield curve is of importance because from it the quantities of the drift term of the local volatility interbank offered rate model can be obtained thus the spot process equation can be accurately calculated.

Table 2. Comparison of Black's model and IBOR model.

\begin{tabular}{lll}
\hline ATM & Black's model & IBOR model \\
\hline Relative error & $89.97 \%$ & $98.22 \%$ \\
\hline
\end{tabular}

The Local volatility Interbank offered rate model is benchmarked using the Black's model for pricing interest rate derivatives. Before pricing the caplet for the developing markets, the model is used to price for the developed markets and its prices compared to the Black's model. From table 2, a comparison is made between the industries accepted black's model for pricing the European caplet and the local volatility IBOR model to ascertain which of the two models marks to market data more efficiently. The finding are that the model prices interest rate options with an accuracy of $98.22 \%$ in the developed markets when marked to market compared to the Black's model. The local volatility IBOR model marks to market more efficiently. As a result of this the local volatility IBOR model is used to price for developing markets.

\section{Conclusion}

In conclusion, the dynamics of the developing markets IBOR rates and those of the developed markets IBOR rates are found to be similar though the developing markets are more volatile. This shows that a model used to price derivatives in a developed market can still be used to price derivatives for developing markets. The local volatility IBOR model is found to be efficient to price the interest rate caplet for developed markets. The model matches option prices in developing markets very well hence its use to price for developed markets. The obtained kolmogorov backward PDE can be used to price interest rate derivatives in markets that exhibit the volatility smile in the long run. When benchmarked with the black's model, the local volatility model produces less errors.

\section{References}

[1] Andersen, L., \& Andreasen, J. (2000). Volatility skews and extensions of the libor market model. Applied Mathematical Finance, 7 (1), 1-32.

[2] Berestycki, H., Busca, J., \& Florent, I. (2002). Asymptotics and calibration of local volatility models. Quantitative finance, 2 (1), 61-69.

[3] Black, F., \& Scholes, M. (1973). The pricing of options and corporate liabilities.

[4] Dupire, B., et al. (1994). Pricing with a smile. Risk, 7 (1), 1820.

[5] Henry-Labordere, P. (2009). Calibration of local stochastic volatility models to market smiles: A monte-carlo approach. Risk Magazine, September.

[6] Heston, S. L. (1993). A closed-form solution for options with stochastic volatility with applications to bond and currency options. Review of financial studies, 6 (2), 327-343.

[7] Hull, J., \& White, A. (1987). The pricing of options on assets with stochastic volatilities. The journal of finance, 42 (2), 281-300.

[8] Jamshidian, F. (1997). Libor and swap market models and measures. Finance and Stochastics, 1 (4), 293-330.

[9] Sepp, A. (2002). Pricing barrier options under local volatility. Math. Comp. 
[10] Stein, E. M., \& Stein, J. C. (1991). Stock price distributions with stochastic volatility: an analytic approach. Review of financial Studies, 4 (4), 727-752.

[11] Wang, D. L, Huixia Judy "Estimation of high conditional quantiles for heavy-tailed distributions", 2012.

[12] Zhu, D., \& Qu, D. (2016). Libor local volatility model: A new interest rate smile model. Wilmott, 2016 (82), 78-87.

\section{Biography}

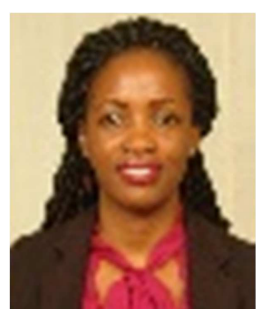

Winnie Chacha received the Bsc. in Financial Engineering and Msc. degrees in Applied Statistics from Jomo Kenyatta University of Agriculture and Technology in 2013 and 2015, respectively. 